\title{
Genetic Variability and Structure of Canadian Populations of the Sapstain Fungus Ceratocystis resinifera
}

\author{
Chantal Morin, Colette Breuil, and Louis Bernier
}

First and third authors: Centre de recherche en biologie forestière, Université Laval, Cité universitaire, Québec G1K 7P4, Canada; and second author: Department of Wood Science, University of British Columbia, 2424 Main Mall, Vancouver, British Columbia V6T 1Z4, Canada. Accepted for publication 14 July 2004.

\begin{abstract}
Morin, C., Breuil, C., and Bernier, L. 2004. Genetic variability and structure of Canadian populations of the sapstain fungus Ceratocystis resinifera. Phytopathology 94:1323-1330.

Genomic DNA was extracted from 129 isolates of Ceratocystis resinifera, a species belonging to the $C$. coerulescens complex, and 19 polymorphic random amplified polymorphic DNA markers were used to study the population genetic structure of this fungus. The analysis suggested a moderate value for genetic diversity $\left(H_{S}=0.209\right)$. However, when monomorphic markers and rare alleles, representing 89 markers, also were included in the calculation, the genetic diversity of Canadian populations of C. resinifera appeared to be much lower $\left(H_{S}=0.045\right)$. This could be explained by two hypotheses: (i) recent introduction of this species into North America and (ii) clonal reproduction (by selfing). No specialization

differentiation index between isolates sampled from Pinus and Picea spp. and on phylogenetic analysis using Dice coefficient of association. In spite of a low genetic diversity, a very high genetic differentiation was observed among the nine geographical populations studied $\left(F_{S T}=20.8 \%\right)$. The genetic differences were especially striking when populations from Eastern Canada were compared with populations from Western Canada $\left(\Phi_{S T}=0.27 \% ; P<0.001\right)$, suggesting that a geographic reproductive barrier occurs in Central Canada. This barrier may be the consequence of a weak migration of insect vectors of $C$. resinifera due to reduced presence of hosts in the Canadian Great Plains, where extensive agriculture occurs. However, results from pairwise $F_{S T}$ matrix and phylogeny of haplotypes suggest that the barrier is not totally impenetrable because some gene flow occurred from the west and from the east in the Big River (Saskatchewan) population located in the middle of the Great Plains.
\end{abstract} by $C$. resinifera for coniferous tree species was observed based on genetic
Sapstain is caused mainly by ophiostomatoid fungi, including species of Ceratocystis, Ceratocystiopsis, Ophiostoma, and Leptographium, which produce melanized hyphae resulting in a dark pigmentation. Although ophiostomatoid fungi generally do not alter the structural properties of wood $(24,26)$, the forest industry nevertheless suffers considerable financial losses due to fungal stains because this aesthetic problem considerably reduces wood value and limits exports to countries with high quality standards. Legislated restrictions about the use of chemical preservatives in the forestry sector, particularly at the timber harvest sites, currently are forcing the lumber industry to find alternative means to control sapstain.

The development and application of spontaneous albino mutant strains derived from otherwise pigmented ophiostomatoid fungi is a promising biological control strategy against sapstain of wood. In a small-scale field study, Behrendt et al. (2) succeeded in controlling sapstain fungi on red pine (Pinus resinosa) logs by using a nonpigmented strain of Ophiostoma piliferum, commercially available under the trade name Cartapip-97, which usually is used to eliminate pitch or wood extractives in pulping processes in paper mills (5). Cartapip significantly reduced the percent of colonization by wild-type Ophiostoma spp. but did not completely exclude them from colonizing some parts of the wood.

In order to be efficient, a control agent should be an aggressive colonizer of wood to prevent the development of other fungi. Fleet $(6,7)$ recently observed that several species of Ophiostoma, including $O$. piliferum, from which Cartapip was developed, invaded principally the bark and the superficial layer of sapwood.

Corresponding author: L. Bernier; E-mail address: Louis.bernier@rsvs.ulaval.ca

Publication no. P-2004-0927-01R

(C) 2004 The American Phytopathological Society
Thus, in this study, Ophiostoma spp. did not colonize logs as deeply as other ophiostomatoid fungi, such as Ceratocystis resinifera Harrington \& Wingfield sp. nov., one of seven known species of Ceratocystis that occur primarily on conifers (14). In laboratory tests carried out on lodge pole pine $(P$. contorta) billets, $C$. resinifera also was found to grow longitudinally seven times faster than $O$. piliferum and 12 times faster than $O$. piceae $(6,7)$. Therefore, an albino strain derived from $C$. resinifera could potentially prevent sapstain better than Ophiostoma albino strains. Thus, we are investigating whether albino strains of $C$. resinifera can be used for the biological control of deep stain in Canadian softwood logs, given the rapid growth of this species on conifer wood and its ability to penetrate deep into the sapwood (6). Moreover, this species was the member of the $C$. coerulescens complex which was the most frequently isolated from softwood logs in a recent survey throughout Canada (18).

The development of a biological control agent derived from C. resinifera should include an assessment of the genetic variability and population structure of this fungus. This type of study, which is required for registration issues in countries such as Canada, also would increase our knowledge about genetic diversity on sapstain fungi and help to prevent the introduction of new genetic material into local populations, if the latter were found to be genetically distinct. Essentially, C. resinifera is a haploid organism which can reproduce both sexually and asexually, by perithecia and conidia typical of the genus Chalara, respectively. The MAT-2 strains are capable of selfing because of unidirectional mating type switching (12). Harrington et al. (13) previously used nuclear and mitochondrial markers to compare genetic diversity among three different species of Ceratocystis differing in their reproductive strategies (outcrossing, selfing, and asexual); however, to our knowledge, the literature does not mention any genetic study of intraspecific population structure for any member of the 
C. coerulescens complex. Thus, the objective of the study described herein was to estimate the levels of genetic diversity and genetic differentiation of the Canadian population of $C$. resinifera. This was achieved by analyzing random amplified polymorphic DNA (RAPD) polymorphisms in 129 isolates from nine sites distributed over six provinces.

\section{MATERIALS AND METHODS}

Sampling and identification of Ceratocystis spp. In summer 2000, freshly cut logs of different coniferous species were stacked in seven lumber mills and in two experimental forests located across Canada (Fig. 1), according to a methodology described previously (28). Western locations included sites in British Columbia, Alberta, and Saskatchewan, whereas sites in Ontario, Québec, and New Brunswick represented eastern locations. Logs were sampled 6 to 8 weeks later. Five disks evenly spaced along the logs were obtained from each cut log crosscut by a chainsaw. The disks were put in individual bags and carried to the laboratory, where they were washed and wrapped in newspaper. The disks then were incubated at $\approx 20^{\circ} \mathrm{C}$ for 5 days. Fungal hyphae and, principally, perithecia of Ceratocystis spp. were aseptically

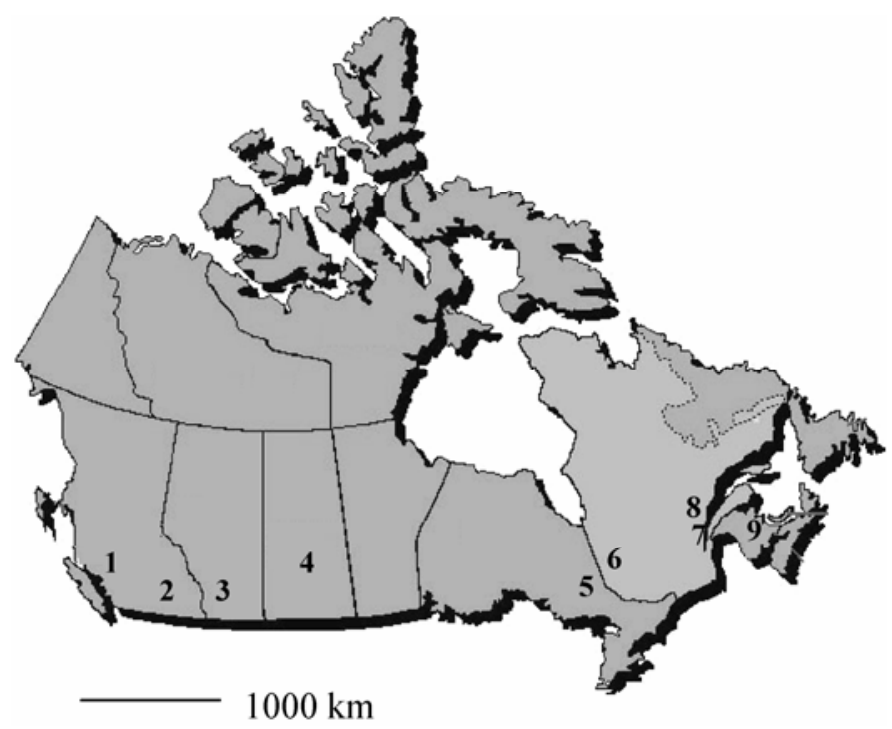

Fig. 1. Sampling sites of Ceratocystis resinifera across Canada. 1, Alex Fraser Forest (British Columbia); 2, Cranbrook (British Columbia); 3, Edson (Alberta); 4, Big River (Saskatchewan); 5, Kirkland Lake (Ontario); 6, LaSarre (Québec); 7, St-Émile (Québec); 8, Forêt Montmorency (Québec); and 9, Plaster Rock (New Brunswick). removed from stained sapwood and transferred onto petri plates containing malt extract agar (MEA) (Oxoid, Nepean, Canada) amended with $0.01 \%$ copper sulphate $\left(\mathrm{CuSO}_{4}\right)$ to inhibit yeast growth. Colonies showing hyphal morphology typical of Ceratocystis spp. were purified to a single spore culture and identified by polymerase chain reaction (PCR) restriction fragment length polymorphism of a $\beta$-tubulin gene fragment (17). Once identification was completed, 15 isolates of $C$. resinifera, the species most frequently isolated, were retained for each site except Cranbrook (British Columbia), from which only 9 isolates of $C$. resinifera were recovered (Table 1). Whenever possible, isolates from the same disk were excluded in the selection, in order to avoid collecting clones. Thus, a total of 129 isolates from nine locations was further analyzed.

DNA extraction and PCR amplification. DNA from each isolate was extracted using a method developed by Kim et al. (16) which was modified by adding a phenol chloroform extraction step prior to isopropanol precipitation. The concentration of extracted DNA was estimated by comparing the band intensity of samples on agarose gel $(1.5 \%)$ with a known amount of lambda DNA standard. In all, 120 RAPD decameric primers (Operon Technologies, Alameda, CA) were screened using nine individuals, one from each of the locations sampled. Primers generating more reproducible and clear polymorphic markers were retained for the amplification of all 129 isolates. DNA was amplified as described previously by Gagné et al. (8). All amplifications were done at least twice to ensure reproducibility of results.

Data analysis. A binary matrix was constructed in which polymorphic markers were scored as putative loci with two alleles, one allele indicating the presence and the other the absence of the marker. Due to the ability of $C$. resinifera to self (12), the segregation of RAPD polymorphisms among sexual progeny was not tested. However, markers which were mutually exclusive (when one marker is never present with one another and vice versa) were eliminated to prevent sampling of multiple alleles of the same locus. A phylogenetic analysis of the haplotypes, based on the genetic distances of Nei-Li (23), was carried out by neighborjoining analysis using PAUP 4.0b10 software (D. L. Swofford, Florida State University). The tree resulting from this analysis as well as the distribution of all haplotypes were translated graphically as a network, as described previously by Hamelin et al. (11), in which the genetic relationships between haplotypes are shown by lines whose length represents the genetic distance. For all remaining analyses, a clone-corrected data set was used due to the presence of multiple clonal genotypes within populations. Allelic frequency, Nei's (22) unbiased heterozygosity $\left(H_{S}\right)$, and total genetic heterozygosity $\left(H_{T}\right)$ were calculated for each locus, after which Wright's genetic differentiation index $\left(F_{S T}\right)$ was estimated

TABLE 1. Locations and wood species sampled across Canada

\begin{tabular}{llr}
\hline Site & \multicolumn{1}{c}{ Host species } & Number of isolates \\
\hline 1. Alex Fraser Forest (British Columbia) & Picea glauca $\times$ P. engelmannii hybrid & 15 \\
2. Cranbrook (British Columbia) & Pinus contorta & 9 \\
3. Edson (Alberta) & P. glauca & 9 \\
& P. contorta & 5 \\
4. Big River (Saskatchewan) & Picea mariana & 1 \\
& P. glauca & 8 \\
5. Kirkland Lake (Ontario) & Pinus banksiana & 5 \\
6. LaSarre (Québec) & P. banksiana & 2 \\
& P. glauca & 14 \\
7. St-Émile (Québec) & P. banksiana & 1 \\
& P. mariana & 11 \\
8. Forêt Montmorency (Québec) & Abies balsamea & 4 \\
9. Plaster Rock (New Brunswick) & P. banksiana & 7 \\
& P. glauca & 6 \\
& P. glauca & 2 \\
& P. mariana & 15 \\
\end{tabular}


among populations, using Weir and Cockerham's methods (30). The heterogeneity of allele frequencies was determined by an exact Fisher test performed by the Tools for Population Genetic Analysis (TFPGA) software (version 1.3; M. Miller, Northern Arizona University, Flagstaff). An analysis of molecular variance (AMOVA) was performed locus by locus, using Arlequin software (version 2.0; Excoffier et al., Geneva University, Switzerland) to partition genetic variability into hierarchical components (within populations, among populations, within groups of populations, and among groups), and to measure the genetic differentiation by $F$ statistic analogs ( $\Phi$ statistics). With this analysis, a matrix of population pairwise $F_{S T}$ also was obtained (29). An AMOVA also was carried out on data from the Québec, Ontario, and New Brunswick populations to determine whether isolates from $P$. banksiana were genetically differentiated from those recovered from Picea spp. Nei's unbiased genetic distances (22) were measured between all pairs of populations and relationships between populations were investigated by constructing a phylogenetic tree with the phenetic approach of the unweighted pair group method with arithmetic average (UPGMA) using the TFPGA software. A Mantel test also was performed with TFPGA to determine whether genetic and geographic distances were correlated. To validate the UPGMA analysis and estimate hidden population substructure of the nine Canadian populations of $C$. resinifera sampled, a Bayesian approach (3) was used with the Bayesian Analysis Population Structure (BAPS) software (version 2.0; Corander et al., Rolf Nevanlinna Institute, University of Helsinki, Finland). This approach considers two or more populations connected by a considerable gene flow as a panmictic population with a certain probability based on marker data and prior geographical information. To visualize the relationships among all fungal isolates, a phylogenetic tree was constructed with NTSYS software (version 2.0; Exeter Software, Setauket, NY) using the Dice index of association (4). Gametic linkage disequilibrium was estimated within groups of populations which were not significantly differentiated, for both the entire and the clone corrected data sets, with an exact test based on the Fisher exact probability test on contingency tables (27) performed by Arlequin software. The level of clonality versus sexual reproduction was evaluated using the index of multilocus association $I_{A}$, as well as the index of association $r_{d}(20)$. The computation of the latter is less dependent on the number of loci analyzed (1), thus allowing comparisons between different studies. These indices were computed by MultiLocus software (version 1.2; Agapow and Burt, Imperial College at Silwood Park, Ascot, Berkshire, UK) and their significance was tested by comparing the observed value with that expected under the null hypothesis of complete panmixis based on 1,000 randomizations of the sample.

\section{RESULTS}

Genetic polymorphisms. A large number of RAPD primers $(n=120)$ had to be screened in order to obtain enough clear polymorphic markers. The 13 primers selected to amplify genomic DNA in 129 isolates generated 112 reproducible fragments from which only 54 (48\%) were polymorphic. Among these polymorphic fragments, 31 occurred at a frequency less then 0.05 and were considered as rare alleles (15). These markers were excluded from the analysis because the number of isolates per population ( $n=15$, except in Cranbrook where $n=9$ ) was too small to verify unambiguously if these rare alleles were absent from some populations. Four additional markers were excluded from the analysis because they were mutually exclusive and might have represented different alleles of the same locus, rather than different loci. Therefore, only 19 markers were submitted to the statistical analysis. Among these, only one marker (OPP12 ${ }_{852}$ ) was a private allele present at a high frequency in the Plaster Rock (New Brunswick) population (Table 2). However, 16\% (3/19) of the markers
$\left(\mathrm{OPA} 10_{1018}\right.$, OPE12 3714 , and OPP12 ${ }_{852}$ ) were observed only in the eastern populations (Fig. 1; population numbers 5 to 9), whereas two markers $\left(\mathrm{OPP} 9_{1539}\right.$ and $\left.\mathrm{OPQ} 5_{690}\right)$ were observed uniquely in western populations (Fig. 1; population numbers 1 to 4 ). Therefore, $26.3 \%(5 / 19)$ of the markers were specific to either eastern or western isolates. Moreover, four other markers (OPA9 ${ }_{1018}$, OPA $9_{900}$, OPA $9_{442}$, and OPG $16_{2442}$ ) were markedly more prevalent in western or in eastern populations. Homogeneity of allelic frequency among populations was observed for only nine markers (Table 2).

Phylogeny and distribution of haplotypes. A total of 75 different RAPD haplotypes were identified among the 129 isolates studied. Analysis of their distribution revealed that no haplotype was found in both an eastern and a western population (Fig. 2). In the west, $25.0 \%$ of the haplotypes were present in at least two populations compared with only $6.7 \%$ in the east. Among western populations, only Cranbrook (British Columbia) and Edson (Alberta) did not share haplotypes with each other. In the east, the Québec populations from St-Émile and Forêt Montmorency, which were separated by the shortest geographic distance $(52 \mathrm{~km})$, shared two frequent haplotypes with each other but none with other populations. Kirkland Lake (Ontario) was the only population that shared no haplotype with any other Canadian population. The genetically most distant haplotypes, numbers 42 and 32, were from Kirkland Lake and Big River (Saskatchewan), respectively (Nei-Li distance $=0.346$ ). Eastern haplotype number 52 (LaSarre, Québec), was the least distant from western haplotypes, especially haplotypes 16 and 22 found in Edson, Alberta (Nei-Li distances $=0.011$ ). Only a few western haplotypes (numbers 26, 27, 30, and 31), all found in Big River, were related with eastern haplotypes (numbers 52, 61, 58, and 33, respectively). The most prevalent haplotype (number 3) was observed eight times $(6 \%)$ and was present in both Edson and the Alex Fraser Forest (British Columbia).

Heterozygosity and differentiation index. The adjusted expected heterozygoty $\left(H_{S}\right)$ per locus for all populations ranged from 0.082 to 0.429 and averaged 0.209 (Table 2). The $H_{S}$ per population over all polymorphic loci, indicating the level of genetic diversity, ranged from 0.108 to 0.285 and averaged 0.209 , accounting for $79 \%$ of the total genetic diversity $\left(H_{T}=0.275\right)$. In general, lower values of diversity index and percentage of polymorphic loci were observed in the west than in the east. The Plaster Rock population, closely followed by the Big River and Kirkland Lake populations, showed the highest level of $H_{S}$ (0.285). The western populations from Alex Fraser Forest and Cranbrook exhibited the lowest percentage of polymorphic loci (31.6\%). In the east, the Forêt Montmorency population had the lowest percentage of polymorphic loci $(47.4 \%)$, whereas a higher value $(73.7 \%)$ was recorded for the geographically adjacent population sampled in St-Émile. The differentiation population index $\left(F_{S T}\right)$ ranged from 0.003 to 0.735 and averaged 0.208 over all loci, indicating a high genetic differentiation (31).

When all 108 markers (including rare alleles, and monomorphic and polymorphic loci, but excluding the four markers whose allelic status was unclear) were included in the calculation (Table $2)$, the value of $H_{T}$ was reduced more than four times $(0.275$ to 0.061). Similarly, the values of $H_{S}$ per population over all loci dropped markedly and ranged from 0.034 to 0.058 and averaged 0.047. Then, the Forêt Montmorency population showed the lowest $H_{S}$ index (0.034), whereas the Big River, Kirkland Lake, and Plaster Rock populations still showed the highest levels $\left(H_{S}=\right.$ $0.057,0.055$, and 0.058 , respectively).

AMOVA. Results from the AMOVA applied to haplotype data from all nine populations indicated that $79.2 \%(P<0.001)$ of genetic variability was due to differences among individual isolates (Table 3). A relatively high proportion of the genetic variability was attributable to differences among populations $\left(20.8 \% ; \Phi_{S T}=\right.$ $0.028 ; P<0.001)$. When populations were assorted into two geo- 
graphically distinct groups, western and eastern, the AMOVA revealed that the proportion of variance due to differences between the two groups was $27.4 \%\left(\Phi_{S T}=0.274 ; P<0.001\right)$ compared with $2.9 \%(P<0.001)$ of variance found among populations within groups. When only western or eastern populations were included in the analysis, differentiation indices were reduced to $0.032(P=$ $0.090)$ and $0.042(P=0.028)$, respectively. The genetic differentiation observed between isolates sampled on $P$. banksiana and those sampled on Picea spp. was low $\left(\Phi_{S T}=0.034\right)(P=0.056)$ (Table 4).

Comparisons of pairs of population samples. Analysis of the matrix of population pairwise $F_{S T}$ indices generated by the AMOVA suggested the occurrence of regional trends (Table 5). Thus, when the four western populations were mutually compared, the observed level of $F_{S T}$ indices was low ( -0.023 to 0.064$)$ and not significant $(P>0.05)$. Genetic distances indices increased markedly (0.111 to 0.457$)$ and were all significant when western populations were compared with eastern populations. Pairwise comparisons among eastern populations resulted in $F_{S T}$ indices slightly higher $(-0.106$ to 0.121$)$ than those computed for western populations, but only indices observed between Plaster Rock and all other eastern populations, except St-Émile, were significant. Among western populations, the Big River (Saskatchewan) population was the least distant from eastern populations, as shown by $F_{S T}$ indices (0.111 to 0.236$)$ lower than those observed when other western populations were compared with eastern populations (0.222 to 0.457$)$. Besides, the genetic distance index (0.111) between the Big River and St-Émile (Québec) populations was lower than the one observed for Plaster Rock and Forêt Montmorency (0.118) or for Plaster Rock and Kirkland Lake (0.121), three eastern populations. Furthermore, the highest $F_{S T}(0.457)$ was observed between the westernmost population, Alex Fraser
Forest in British Columbia, and the Kirkland Lake population in Ontario. In the west, the largest $F_{S T}$ index (0.064) was observed between the geographically closest populations (Cranbrook and Edson). In the east, the largest genetic distance was observed between Kirkland Lake and the Plaster Rock (New Brunswick) populations (0.121). The latter was the only population in the East presenting significant genetic distances with other eastern populations.

The pairwise $F_{S T}$ followed a similar trend with estimates of Nei's unbiased genetic distances among populations which ranged from -0.007 to 0.229 (data not shown). Moreover, the Mantel test showed that genetic distances were significantly correlated with geographic distances $(r=0.795, P=0.004)$ when the entire data set was analyzed. However, no correlations were found between genetic and geographic distances when the Mantel test was applied to regional subsets of data (west; $r=-0.229, P=0.666$; east; $r=0.378, P=0.058$ ).

Cluster analyses. The UPGMA cluster analysis of Nei's unbiased distances (Fig. 3) confirmed that the eastern populations were genetically different from western ones. Only the corresponding node, which was supported in $100 \%$ of the resampling (1,000 bootstraps), was significant in the dendrogram. This analysis also showed that the Plaster Rock population was the most differentiated from other eastern populations. The scale presented with the UPGMA tree revealed that genetic distances among all populations nevertheless were low (less than 0.12). The Bayesian analysis highly supported the UPGMA dendrogram because the most probable structure $(P=0.6415)$ separated isolates in three different clusters: the first one included all western populations, whereas the second cluster included all eastern populations except Plaster Rock, which made up the third cluster. Finally, the phylogenetic tree based on Dice coefficient of association indicated that

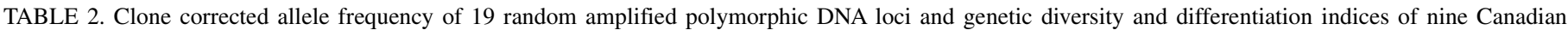
populations of Ceratocystis resinifera

\begin{tabular}{|c|c|c|c|c|c|c|c|c|c|c|c|c|c|}
\hline \multirow[b]{2}{*}{ Locus } & \multicolumn{9}{|c|}{ Allele frequency ${ }^{\mathrm{a}}$} & \multicolumn{4}{|c|}{ Genetic diversity $^{\mathrm{b}}$} \\
\hline & $\mathrm{AF}(12)$ & $\mathrm{C}(7)$ & $\mathrm{E}(11)$ & $\mathrm{BR}(14)$ & $\mathrm{K}(11)$ & $\mathrm{L}(11)$ & SE (8) & FM (7) & PR (10) & $P$ & $H_{S}$ & $H_{T}$ & $F_{S T}$ \\
\hline OPA9 $9_{1018}$ & 1.000 & 1.000 & 1.000 & 0.857 & 0.000 & 0.091 & 0.125 & 0.143 & 0.200 & $* *$ & 0.140 & 0.502 & 0.735 \\
\hline OPA9990 & 0.167 & 0.286 & 0.182 & 0.519 & 1.000 & 1.000 & 0.875 & 1.000 & 1.000 & $* *$ & 0.202 & 0.459 & 0.546 \\
\hline OPA $9_{442}$ & 1.000 & 1.000 & 0.909 & 0.643 & 0.273 & 0.455 & 0.625 & 0.714 & 0.400 & $* *$ & 0.337 & 0.452 & 0.228 \\
\hline OPA 9435 & 0.000 & 0.000 & 0.091 & 0.000 & 0.182 & 0.273 & 0.000 & 0.000 & 0.300 & ns & 0.149 & 0.179 & 0.084 \\
\hline OPA $10_{1018}$ & 0.000 & 0.000 & 0.000 & 0.000 & 0.000 & 0.000 & 0.125 & 0.000 & 0.500 & $* *$ & 0.084 & 0.124 & 0.365 \\
\hline OPA $11_{1259}$ & 0.000 & 0.000 & 0.000 & 0.286 & 0.273 & 0.364 & 0.250 & 0.143 & 0.200 & ns & 0.258 & 0.291 & 0.044 \\
\hline OPE12 3714 & 0.000 & 0.000 & 0.000 & 0.000 & 0.364 & 0.182 & 0.125 & 0.000 & 0.000 & $*$ & 0.114 & 0.143 & 0.155 \\
\hline OPF16 2346 & 0.000 & 0.000 & 0.091 & 0.143 & 0.455 & 0.273 & 0.125 & 0.000 & 0.400 & $*$ & 0.233 & 0.291 & 0.111 \\
\hline OPF16 2036 & 0.917 & 0.857 & 0.818 & 0.786 & 1.000 & 1.000 & 1.000 & 1.000 & 0.900 & ns & 0.143 & 0.161 & 0.003 \\
\hline OPG $16_{2442}$ & 1.000 & 1.000 & 1.000 & 1.000 & 0.636 & 1.000 & 0.875 & 1.000 & 0.800 & $* *$ & 0.117 & 0.143 & 0.162 \\
\hline OPG16 2036 & 0.833 & 1.000 & 0.636 & 0.643 & 0.818 & 0.727 & 0.625 & 0.714 & 0.700 & ns & 0.376 & 0.391 & 0.038 \\
\hline $\mathrm{OPP}_{1539}$ & 0.417 & 0.714 & 0.273 & 0.500 & 0.000 & 0.000 & 0.000 & 0.000 & 0.000 & $* *$ & 0.210 & 0.345 & 0.319 \\
\hline OPP12 1900 & 0.000 & 0.000 & 0.091 & 0.071 & 0.364 & 0.091 & 0.125 & 0.286 & 0.100 & ns & 0.203 & 0.214 & 0.040 \\
\hline OPP12 1766 & 0.750 & 0.571 & 0.909 & 0.714 & 0.546 & 0.818 & 0.875 & 0.714 & 0.900 & ns & 0.359 & 0.369 & 0.007 \\
\hline OPP12 852 & 0.000 & 0.000 & 0.000 & 0.000 & 0.000 & 0.000 & 0.000 & 0.000 & 0.700 & $* *$ & 0.049 & 0.143 & 0.669 \\
\hline $\mathrm{OPQ}_{730}$ & 0.833 & 0.714 & 0.727 & 0.643 & 0.727 & 0.546 & 0.750 & 0.714 & 0.700 & ns & 0.429 & 0.420 & 0.074 \\
\hline OPQ1 $1_{725}$ & 0.000 & 0.000 & 0.182 & 0.000 & 0.091 & 0.091 & 0.125 & 0.143 & 0.200 & ns & 0.166 & 0.161 & 0.023 \\
\hline $\mathrm{OPQ5}_{690}$ & 0.000 & 0.000 & 0.364 & 0.143 & 0.000 & 0.000 & 0.000 & 0.000 & 0.000 & $* *$ & 0.082 & 0.124 & 0.182 \\
\hline OPQ13 653 & 1.000 & 0.857 & 0.818 & 0.786 & 0.636 & 0.727 & 0.750 & 0.714 & 0.900 & ns & 0.317 & 0.319 & 0.021 \\
\hline Average (19 loci) & $\ldots$ & $\ldots$ & $\ldots$ & $\ldots$ & $\ldots$ & $\ldots$ & $\ldots$ & $\ldots$ & $\ldots$ & $\ldots$ & 0.209 & 0.275 & 0.208 \\
\hline Average $(108 \text { loci })^{\mathrm{c}}$ & $\ldots$ & $\ldots$ & $\ldots$ & $\ldots$ & $\ldots$ & $\ldots$ & $\ldots$ & $\ldots$ & $\ldots$ & $\ldots$ & 0.047 & 0.061 & $\ldots$ \\
\hline$H s \mathrm{~b}(19 \text { loci })^{\mathrm{d}}$ & 0.108 & 0.125 & 0.206 & 0.258 & 0.264 & 0.228 & 0.226 & 0.181 & 0.285 & $\ldots$ & $\ldots$ & $\ldots$ & $\ldots$ \\
\hline$H s c(108 \text { loci })^{\mathrm{e}}$ & 0.039 & 0.038 & 0.036 & 0.057 & 0.055 & 0.052 & 0.051 & 0.034 & 0.058 & $\ldots$ & $\ldots$ & $\ldots$ & $\ldots$ \\
\hline Polym. loci (\%) & 31.58 & 31.58 & 68.42 & 68.42 & 63.16 & 63.16 & 73.68 & 47.37 & 78.95 & $\ldots$ & $\ldots$ & $\ldots$ & $\ldots$ \\
\hline
\end{tabular}

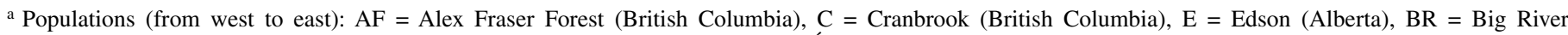
(Saskatchewan), KL = Kirkland Lake (Ontario), L = LaSarre (Québec), SE = St-Émile (Québec), FM = Forêt Montmorency (Québec), and PR = Plaster Rock (New Brunswick); number in parentheses = number of samples/location.

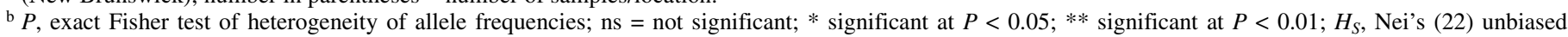
genetic diversity index representing expected heterozygosity of individuals in their population; $H_{T}$, unbiased expected heterozygosity of individuals if the nine populations are considered as a whole population; $F_{S T}$, Wright's genetic differentiation index calculated according to Weir and Cockerham's methods (30).

c Average of $H_{S}$ and $H_{T}$ when rare alleles, and monomorphic and polymorphic loci were included (108 loci).

${ }^{\mathrm{d}} H s \mathrm{~b}$, unbiased genetic diversity index for each population over all polymorphic loci (19 loci).

e $H s c$, unbiased genetic diversity index for each population over all loci when rare alleles, monomorphic and polymorphic loci were included (108 loci). 
most isolates from western Canada were clustered together. A similar trend was observed for eastern isolates. However, no notable association was detected among isolates sampled from the same tree species (data not shown).

Gametic linkage disequilibrium and indices of association. Linkage disequilibrium was evaluated for the entire set of isolates and was positive for a relatively high number of loci $(29.2 \%$; $25.1 \%$ for clone-corrected data) (Table 6). Western and eastern populations were found to differ significantly based on cluster analyses; therefore, linkage disequilibrium also was assessed on the four western populations as a whole, as well as on four of the five eastern populations taken together. The Plaster Rock popula- tion was excluded from the analysis because it was significantly differentiated from the other eastern populations (Table 5; Fig. 3) and contained too few isolates to be considered on its own. In the western group, the percentages of linked loci were similar and moderate whether all isolates or only unique haplotypes (clonecorrected data) were considered (21.9 and $22.9 \%$, respectively) (Table 6). These values were slightly higher than those computed for the eastern group (20\% for all isolates and $17 \%$ for unique haplotypes). The indices of association deviated significantly from the null hypothesis for the western group $\left(I_{A}=0.306\right.$ and $\left.\mathrm{r}_{d}=0.023, P=0.024\right)$ but not for the eastern group for clone-corrected data $\left(I_{A}=0.128\right.$ and $\left.\mathrm{r}_{d}=0.010, P=0.128\right)$. When isolates
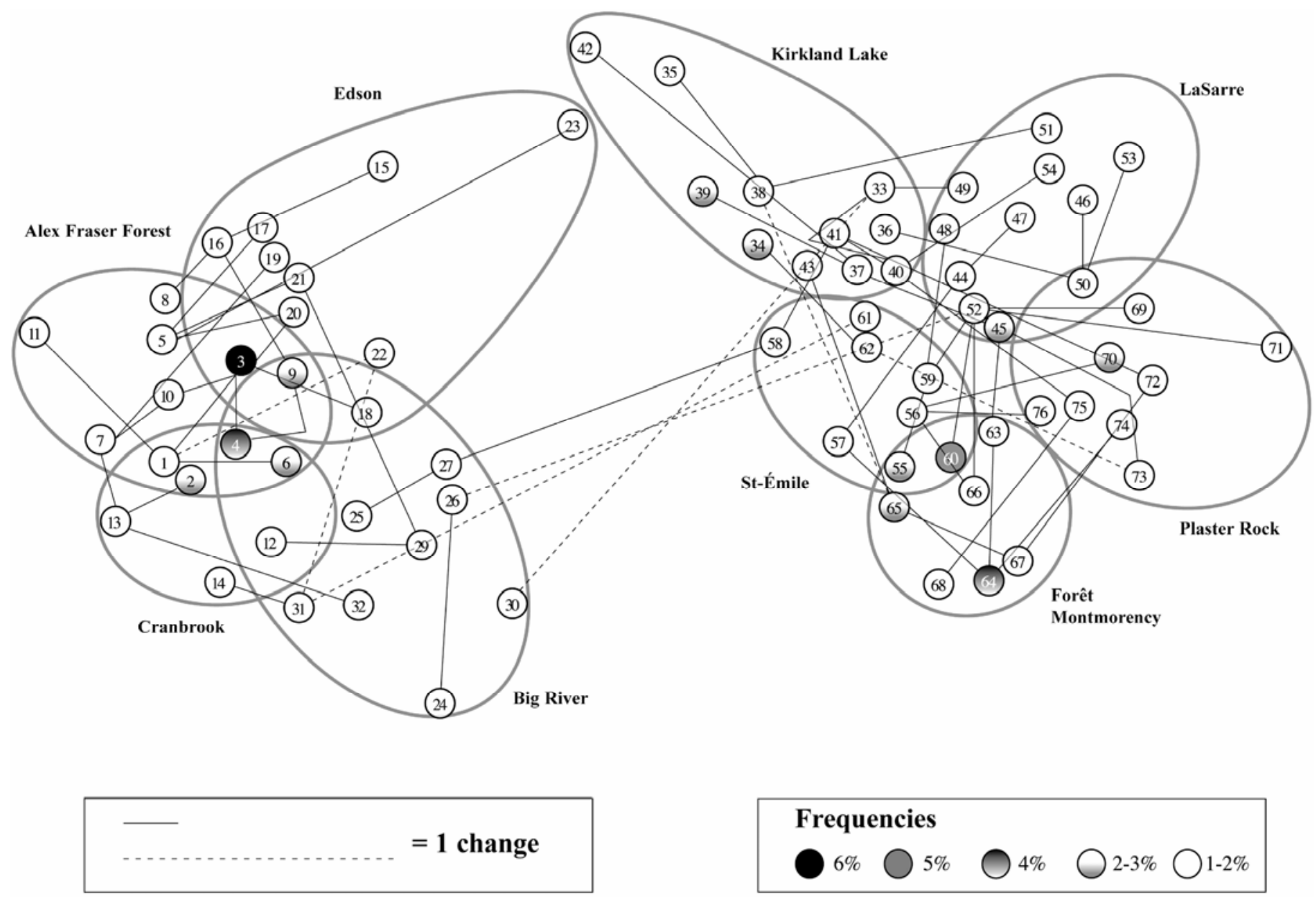

\section{Frequencies

$6 \% \bigcirc 5 \% \bigcirc 4 \% \bigcirc 2-3 \% \bigcirc 1-2 \%$

Fig. 2. Distribution of the 75 different random amplified polymorphic DNA haplotypes detected in 129 Canadian isolates of Ceratocystis resinifera and the neighbor-joining dendrogram based on a matrix of Nei-Li genetic distances between all pairs of haplotypes. Haplotypes are represented as spheres connected by lines whose length corresponds to genetic distance. The frequency of each haplotype is shown by the intensity of the motif of spheres. Because of graphic constraints, genetic distances identified by a dashed line are shown at a different scale than distances identified by a solid line.

TABLE 3. Analysis of molecular variance of random amplified polymorphic DNA markers for 91 haplotypes of Ceratocystis resinifera ${ }^{\mathrm{a}}$

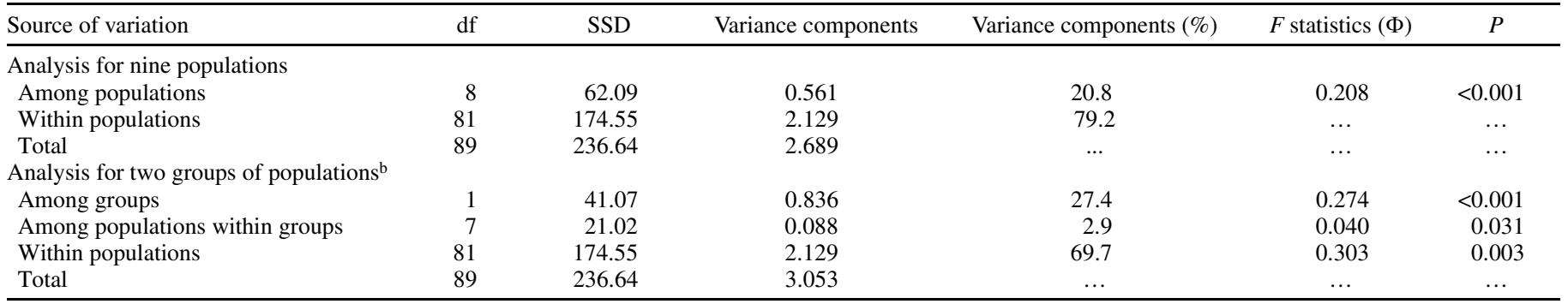

a df, degrees of freedom; SSD, sums of squared deviations; and $P$, probability of obtaining equal or larger value determined by 1,023 permutations.

${ }^{\mathrm{b}}$ Groups were eastern and western Canada. Western Canada = populations from Alex Fraser Forest and Cranbrook (British Columbia), Edson (Alberta), and Big River $($ Saskatchewan). Eastern Canada = populations from Kirkland Lake (Ontario); LaSarre, St-Émile, and Forêt Montmorency (Québec); and Plaster Rock (New Brunswick). 
rather than haplotypes were included in the calculation, the indices of association were significantly different from zero for both groups of populations (western group: $I_{A}=0.608$ and $\mathrm{r}_{d}=0.046$, $P<0.001$; eastern group: $I_{A}=0.265$ and $\left.\mathrm{r}_{d}=0.020, P=0.007\right)$.

\section{DISCUSSION}

The analysis of RAPD polymorphisms in 91 haplotypes representing nine different Canadian populations of $C$. resinifera indicated a level of genetic diversity slightly over $20 \%\left(H_{S}=0.209\right)$. A similar value $(H=0.214)$ was reported for the homothallic species $C$. albofundus, which causes wattle wilt in South Africa, by Roux et al. (25), who concluded that $C$. albofundus exhibited a high level of genetic diversity and was native to South Africa. In the case of $C$. resinifera, however, the value of genetic diversity index probably was overestimated, considering the effort required for selecting primers that generated clear polymorphic fragments. Thus, 120 RAPD primers had to be screened in order to select 13 primers which generated 112 markers but only 19 (17\%) clear polymorphic ones, a much lower result compared with the percentage of polymorphic loci $(94 \%)$ obtained for C. albofundus. Indeed, when monomorphic and polymorphic markers were included in the calculation of the genetic diversity indices for $C$. resinifera, the values decreased by more than four times $\left(H_{S}=0.047\right.$ and $H_{T}=$ $0.061)$, indicating that genetic variability among all individuals studied was, in fact, low. Thus, in this regard, our results suggest that $C$. resinifera could have been introduced in Canada rather than being indigenous, though a comparative analysis of populations from other continents would be required to confirm or invalidate this hypothesis.

TABLE 4. Analysis of molecular variance of random amplified polymorphic DNA markers for two subpopulations of haplotypes of Ceratocystis resinifera sampled from Pinus $(n=21)$ and Picea $(n=22)$ spp. $^{\mathrm{a}}$

\begin{tabular}{lrrrrc}
\hline Source of variation & $\mathrm{df}$ & SSD & Variance components & Variance components $(\%)$ & $F$ statistics $(\Phi)$ \\
\hline Analysis for nine populations & & & & & \\
Among populations & 1 & 4.34 & 0.087 & 3.4 & 0.034 \\
Within populations & 41 & 101.47 & 2.475 & 96.6 & 0.056 \\
Total & 42 & 105.81 & 2.562 & $\ldots$ & $\ldots$ \\
\hline
\end{tabular}

a $\mathrm{df}$, degrees of freedom; SSD, sums of squared deviations; $P$, probability of obtaining equal or larger value determined by 1,023 permutations.

TABLE 5. Ceratocystis resinifera populations pairwise $F_{S T}$ (distance method) from analysis of molecular variance ${ }^{\mathrm{a}}$

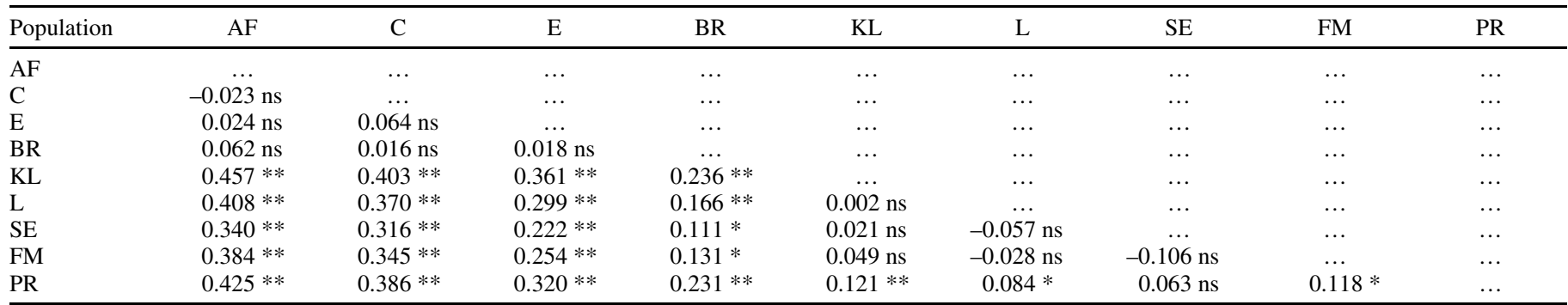

${ }^{a}$ Populations (from west to east): AF = Alex Fraser Forest (British Columbia), $\mathrm{C}=$ Cranbrook (British Columbia), E = Edson $($ Alberta), BR $=$ Big River (Saskatchewan), KL = Kirkland Lake (Ontario), L = LaSarre (Québec), SE = St-Émile (Québec), FM = Forêt Montmorency (Québec), and PR = Plaster Rock (New Brunswick); ns $=$ not significant; * significant at $P<0.05$; ** significant at $P<0.01$.
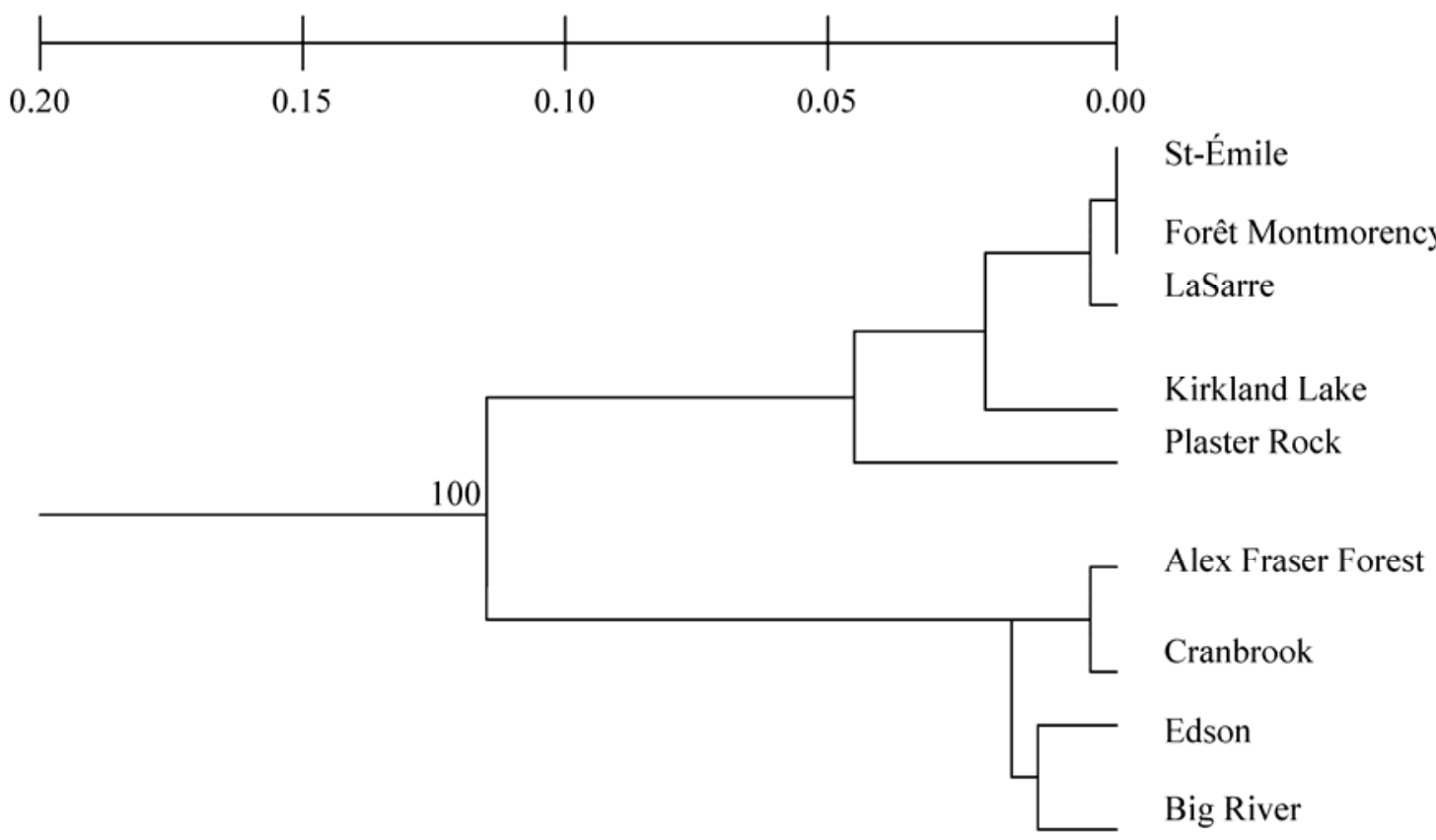

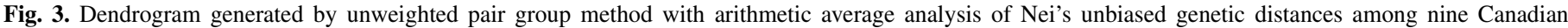

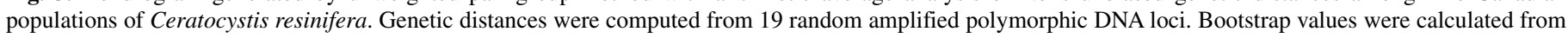
1,000 bootstraps. Only bootstrap values of $50 \%$ or greater are shown. 
C. resinifera reproduces sexually by outcrossing but it also can self by mating type switching (12). This was reflected in the laboratory, because we regularly observed perithecia in single spore cultures, whereas crossing events rarely occurred following pairing of different isolates on solid media (data not shown). This behavior also could explain the low diversity observed in the field because selfing reduces genetic recombination and genetic diversity. This is in concordance with the study of Harrington et al. (13), who examined genetic variation in three Ceratocystis spp., C. eucalypti, C. virescens, and Chalara australis (an anamorph of this genus, without known teleomorph), with three different reproductive strategies: outcrossing, selfing, and asexual reproduction, respectively. These authors noticed an intermediate level of genetic diversity $(H=0.093)$ for the selfing species, $C$. virescens, compared with $H=0.249$ for the outcrossing $C$. eucalypti and $H=0.002$ for the asexually reproducing Chalara australis. Thus, our results $\left(H_{S}=0.047\right)$ suggest that $C$. resinifera also may favor selfing over outcrossing under field conditions. This possibility is supported further by results from the analysis of multilocus associations (Table 6) where indices that deviated significantly from the null hypothesis of panmixis were observed for the group of western populations of $C$. resinifera. Although the corresponding indices were not significant for the eastern group, the probable occurrence of recombination in these populations is not sufficient to reject the hypothesis that clonal reproduction plays an important role in shaping these populations, because it takes relatively low frequencies of recombination to produce all appearances of random mating in a population (21). Moreover, when the analysis of eastern populations was based on isolates rather than haplotypes, the indices of association then were significantly different from zero.

In spite of a low genetic diversity, a high level of genetic differentiation was observed among the nine geographical populations of $C$. resinifera studied, suggesting the occurrence of a barrier to gene flow in Canada. At first glance, results from the Mantel test suggest that this barrier could be the consequence of extensive distances between populations because genetic distances were correlated with geographic distances, indicating that the dispersal was restrained by space. Nevertheless, the correlation between genetic and geographic distances was not significant when only eastern populations or western populations were included in the analysis. Thus, the significant correlation observed from the Mantel test could simply reflect the genetic differentiation between western and eastern populations shown by the UPGMA analysis (Fig. 3). The resulting dendrogram clearly separated eastern populations from western ones and, thus, proposed that the barrier to gene flow occurs in central Canada, between Saskatchewan and Ontario. The matrix of pairwise $F_{S T}$ (Table 4) also suggested that the barrier to gene flow was situated in this area. Interestingly, these results differ to some extent from those reported previously by Gagné et al. (8), who analyzed RAPD polymorphisms in populations of another sapstain fungus, O. piceae, from seven Canadian locations that included four sites (Big River, Kirkland Lake, St-Émile, and Plaster Rock) that also were sampled in the present study. There was no clear evidence that a barrier to gene flow occurred among populations of $O$. piceae. Moreover, populations from Saskatchewan (Big River) and Ontario (Kirkland Lake) were genetically closer to each other than to remaining populations located west or east of these two locations. The reason for this apparent difference between the populations of these two ophiostomatoid species remains unknown.

A barrier to gene flow similar to the one that seems to be operating in $C$. resinifera already has been observed between eastern and western populations of Cronartium ribicola in North America (10). This observation was explained by the absence or patchiness of host and by the development of extensive agriculture across the Great Plains. C. resinifera is dispersed mainly by nonspecific insect vectors such as arthropods, sap-feeding insects and, more rarely, bark beetles $(9,14,19)$. These vectors probably migrate scarcely across the Great Plains due to the reduced presence of hosts; thus, this different ecozone really could be a barrier to gene flow. In the present study, the Saskatchewan population, located in central Canada, was clustered with western populations but was the least differentiated from the eastern populations. It also was one of the populations that showed the highest levels of diversity. This suggests that, although appreciable gene flow occurred between the Big River and other western populations, limited yet detectable gene flow also took place between the former and populations in eastern Canada. Thus, the Canadian Plains do not seem to restrict gene flow completely, probably in part because it could be counteracted by movements of logs and wood products throughout the country.

The phylogeny and distribution of haplotypes also was in concordance with the genetic differentiation observed between eastern and western populations, because no haplotype was shared between these two groups of populations and very few western haplotypes were genetically related to eastern haplotypes. In this analysis, the Kirkland Lake (Ontario) population appeared more genetically different due to the presence of the most distant haplotype (number 42) and the absence of shared haplotypes, whereas AMOVA (pairwise $F_{S T}$ indices), UPGMA, and Bayesian analyses showed that the Plaster Rock population was the most differentiated of the eastern populations. The phylogeny further supported results from other analyses suggesting that the Big River (Saskatchewan) population might act as a link between eastern and western populations, because it was the only western population that contained haplotypes which appeared to be related to eastern haplotypes. Moreover, results from the above analyses suggest that western populations either rely more on clonal reproduction or evolved more recently than eastern populations, because the former exhibited lower genetic diversity and included several haplotypes which occurred in several copies and in at least two locations.

A second, less-pronounced barrier to gene flow in Canadian populations of $C$. resinifera was detected by the Bayesian analysis and AMOVA (pairwise $F_{S T}$ indices) between the Plaster Rock population and the other eastern populations. The occurrence of this barrier cannot be explained by the mechanisms described

TABLE 6. Gametic linkage disequilibrium and indices of multilocus association $\left(I_{A}\right.$ and $\left.\mathrm{r}_{d}\right)$ in Canadian populations of Ceratocystis resinifera

\begin{tabular}{|c|c|c|c|c|c|c|c|c|c|c|}
\hline \multirow[b]{2}{*}{ Population $^{\mathrm{a}}$} & \multirow[b]{2}{*}{ Polym. loci } & \multirow[b]{2}{*}{ Comparisons } & \multicolumn{4}{|c|}{ Isolates $^{\mathrm{b}}$} & \multicolumn{4}{|c|}{ Haplotypes ${ }^{\mathrm{b}}$} \\
\hline & & & LD (\%) & $I_{A}$ & $\mathrm{r}_{d}$ & $n$ & $\mathrm{LD}(\%)$ & $I_{A}$ & $r_{\mathrm{d}}$ & $n$ \\
\hline All nine populations & 19 & 171 & 29.2 & $\cdots$ & $\ldots$ & 129 & 25.1 & $\ldots$ & $\ldots$ & 91 \\
\hline Western Canada & 15 & 105 & 21.9 & $0.608 * * *$ & $0.046 * * *$ & 54 & 22.9 & $0.306^{*}$ & $0.023 *$ & 32 \\
\hline Eastern Canada & 15 & 105 & 20.0 & $0.265^{* *}$ & $0.020 * *$ & 60 & 17.1 & 0.128 & 0.010 & 35 \\
\hline
\end{tabular}

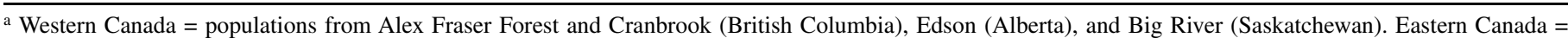
populations from Kirkland Lake (Ontario); and LaSarre, St-Émile, and Forêt Montmorency (Québec).

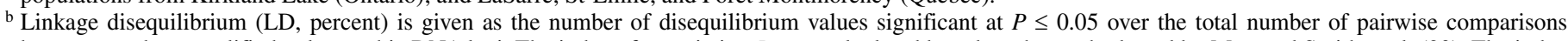
between random amplified polymorphic DNA loci. The index of association $I_{A}$ was calculated based on the method used by Maynard Smith et al. (20). The index of association $r_{d}$ was calculated using the method developed by Agapow and Burt (1). The significance of the both indices was tested by 1,000 randomizations. Significance levels: $* * *, * *$, and $*=P<0.001,0.01$, and 0.05 , respectively. 
above. If the hypothesis of a recent introduction of $C$. resinifera into Canada is favored, the genetic differentiation observed between Plaster Rock and other eastern populations could then reflect the occurrence of two distinct introductions. Because the LaSarre and Plaster Rock populations shared one haplotype (Fig. 2 ), it seems that migration occurs and eventually could lead to a decrease in the genetic differentiation observed.

Fungal isolates were not always recovered from the same tree species in western and in eastern Canada. Thus, the differentiation between western and eastern populations might be explained, in part, by an adaptation for different tree species. In their original description of $C$. resinifera, Harrington and Wingfield (14) mentioned that it had, until then, been found only on Picea spp., suggesting host specialization. However, in the survey we conducted across Canada, $C$. resinifera also was recovered from pine logs, as well as from balsam fir logs $(17,18)$. Analysis of the phylogenetic tree based on Dice coefficient revealed no significant association among isolates sampled on the same tree species. In addition, the $F_{S T}$ index computed from the AMOVA suggested that genetic differentiation between isolates sampled from Pinus banksiana and from Picea spp. was low. Thus, our results indicate that the host range of $C$. resinifera is broader than previously thought and further suggest that isolates from different hosts belong to the same genetic entity. However, because our survey did not provide isolates for all tree species studied in each sampling site, further investigations are needed to confirm or invalidate the possible occurrence of specialization for host species. Moreover, neutral markers such as the RAPDs used in this study are poorly suited for detecting physiological adaptation, because they do not necessarily represent DNA coding regions.

In spite of a high genetic differentiation observed among western and eastern populations, additional work is needed to unambiguously conclude to the presence of two different biotypes of C. resinifera in Canada. Genetic diversity among all isolates was relatively small, as revealed by the high number of monomorphic RAPD fragments observed and the low value of $H_{S}$ and $H_{T}$ when calculations included all markers. Moreover, the results of this study also showed that the barrier is not totally impenetrable to gene flow. Therefore, there appears to be a low probability of disturbing local populations by the application of one native, albino isolate of $C$. resinifera used as a biocontrol agent across Canada. Otherwise, further studies are necessary to verify the absence of pathogenicity in $C$. resinifera.

\section{ACKNOWLEDGMENTS}

Funding for this work was provided by a Natural Sciences and Engineering Research Council of Canada (NSERC) Strategic grant to L. Bernier and C. Breuil. We wish to thank personnel at the participating mills and experimental forests; D. Gravel, E. Leclerc, P. Loppnau, and G. Racine for their help in the sampling, isolation, and molecular study of C. resinifera; and N. Feau, P. Tanguay, and two reviewers for critical comments of earlier versions of the manuscript.

\section{LITERATURE CITED}

1. Agapow, P. M., and Burt, A. 2001. Indices of multilocus linkage disequilibrium. Mol. Ecol. Notes 1:101-102.

2. Behrendt, C. J., Blanchette, R. A., and Farrell, R. L. 1995. Biological control of blue stain fungi in wood. Phytopathology 85:92-97.

3. Corander, J., Waldmann, P., and Sillanpää, M. J. 2003. Bayesian analysis of genetic differentiation between populations. Genetics 163:367-374.

4. Dice, L. R. 1945. Measures of the amount of ecologic association between species. Ecology 26:297-302.

5. Farrell, R. L., Blanchette, R. A., Brush, T. S., Hadar, Y., Iverson, S., Krisa, K., Wendler, P. A., and Zimmerman, W. 1993. Cartapip: A biopulping product for control of pitch and resin acid problems in pulp mills. J. Biotechnol. 30:115-122.
6. Fleet, C. A. 2001. Growth, nutrition and genetic factors that affect pigmentation of wood-sapstain fungi. Master's thesis, Department of Wood Sciences, Faculty of Forestry, University of British Columbia, Vancouver, Canada.

7. Fleet, C. A., Breuil, C., and Uzunovic, A. 2001. Nutrient consumption and pigmentation of deep and surface colonizing sapstaining fungi in Pinus contorta. Holzforschung 55:340-346.

8. Gagné, P., Yang D.-Q., Hamelin, R. C., and Bernier, L. 2001. Genetic Variability of Canadian populations of the sapstain fungus Ophiostoma piceae. Phytopathology 91:369-376.

9. Gibbs, J. N. 1993. The biology of ophiostomatoid fungi causing sapstain in trees and freshly cut logs. Pages 153-160 in: Ceratocystis and Ophiostoma. Taxonomy, Ecology and Pathogenicity. M. J. Wingfield, K. A. Seifert, and J. F. Webber, eds. The American Phytopathological Society, St. Paul, MN.

10. Hamelin, R. C., Hunt, R. S., Geils, B. W., Jensen, G. D., Jakobi, V., and Lecours, N. 2000. Barrier to gene flow between eastern and western populations of Cronartium ribicola in North America. Phytopathology 90:1073-1078.

11. Hamelin, R. C., Lecours, N., and Laflamme, G. 1998. Molecular evidence of distinct introductions of the European race of Gremmeniella abietina into North America. Phytopathology 88:582-588.

12. Harrington, T. C., and McNew, D. L. 1997. Self-fertility and uni-directional mating-type switching in Ceratocystis coerulescens, a filamentous ascomycete. Curr. Genet. 32:52-59.

13. Harrington, T. C., Steimel, J., and Kile, G. 1998. Genetic variation in three Ceratocystis species with outcrossing, selfing and asexual reproductive strategies. Eur. J. For. Pathol. 28:217-226.

14. Harrington, T. C., and Wingfield, M. J. 1998. The Ceratocystis species on conifers. Can. J. Bot. 76:1446-1457.

15. Hartl, D. L., and Clark, A. G. 1997. Genetic and phenotypic variation. Pages 53-54 in: Principles of Population Genetics, 3rd ed. Sinauer Associates, Inc., Sunderland, MA.

16. Kim, S. H., Uzunovic, A., and Breuil, C. 1999. Rapid detection of Ophiostoma piceae and O. quercus in stained wood by PCR. Appl. Environ. Microbiol. 65:287-290.

17. Loppnau, P., and Breuil, C. 2003. Species level identification of conifer associated Ceratocystis sapstain fungi by PCR-RFLP on a $\beta$-tubulin gene fragment. FEMS Microbiol. Lett. 222:143-147.

18. Loppnau, P., Morin, C., Kim, S. H., Breuil, C., and Bernier, L. 2001. Ceratocystis species causing deep stain in Canadian softwood logs. (Abstr.) Phytopathology 91(suppl.):S56.

19. Malloch, D., and Blackwell, M. 1993. Dispersal biology of the ophiostomatoid fungi. Pages 195-206 in: Ceratocystis and Ophiostoma. Taxonomy, Ecology and Pathogenicity. M. J. Wingfield, K. A. Seifert, and J. F. Webber, eds. The American Phytopathological Society, St. Paul, MN.

20. Maynard Smith, J., Smith, N. H., O'Rourke, M., and Spratt, B. G. 1993. How clonal are bacteria? Proc. Natl. Acad. Sci. USA 90:4384-4388.

21. Milgroom, G. M. 1996. Recombination and the multilocus structure of fungal populations. Annu. Rev. Phytopathol. 34:457-477.

22. Nei, M. 1978. Estimation of average heterozygosities and genetic distance from a small number of individuals. Genetics 89:583-590.

23. Nei, M., and Li, W.-H. 1979. Mathematical model for studying genetic variation in terms of restriction endonucleases. Proc. Natl. Acad. Sci. USA 76:5269-5273.

24. Roff, J. W., Cserjesi, A. J., and Swann, H. W. 1974. Prevention of sapstain and mold in packaged lumber. Can. For. Serv. Publ. 1325.

25. Roux, J., Harrington, T. C., Steimel, J. P., and Wingfield, M. J. 2001. Genetic variation in the wattle wilt pathogen Ceratocystis albofundus. Mycoscience 42:327-332.

26. Seifert, K. A. 1993. Sapstain of commercial lumber by species of Ophiostoma and Ceratocystis. Pages 141-151 in: Ceratocystis and Ophiostoma. Taxonomy, Ecology and Pathogenicity. M. J. Wingfield, K. A. Seifert, and J. F. Webber, eds. The American Phytopathological Society, St. Paul, MN.

27. Slatkin, M. 1994. Linkage disequilibrium in growing and stable populations. Genetics 137:331-336.

28. Uzunovic, A., Yang, D.-Q., Gagné, P., Breuil, C., Bernier, L., Byrne, A., Gignac, M., and Kim, S. H. 1999. Fungi that cause sapstain in Canadian softwoods. Can. J. Microbiol. 45:914-922.

29. Weir, B. S. 1996. Genetic Data Analysis II: Methods for Discrete Population Genetic Data. Sinauer Associates, Inc., Sunderland, MA.

30. Weir, B. S., and Cockerham, C. C. 1984. Estimating F-statistics for the analysis of population structure. Evolution 38:1358-1370.

31. Wright, S. 1978. Evolution and the Genetics of Populations, vol. 4. Variability Within and Among Natural Populations. University of Chicago Press, Chicago. 